\title{
Determination of Total Volatile Basic Nitrogen (TVB-N) Content in Beef by Hyperspectral Imaging Technique
}

\author{
Shanmei Liu ${ }^{1}, \mathrm{Lu} \mathrm{Gan}^{2, \mathrm{a}}$ \\ ${ }^{1}$ College of Informatics, Huazhong Agricultural University, Wuhan 430070,China; \\ ${ }^{2}$ School of Electric and Electronic Engineering, Wuhan Polytechnic University, Wuhan 430023,china;
}

\begin{abstract}
Non-destructive determination of TVB-N content in beef using hyperspectral imaging (HSI) technique was evaluated. In order to create a robust model to predict the TVB-N content in beef, partition of sample set, spectral pretreatment, and the optimum wavelength selection were discussed. After the beef sample set was parted by concentration gradient (CG) algortithm, and the spectra of beef samples were preprocessed by standard normalized variate (SNV) combined with auto scale(AS), the partial least square regression (PLSR) model was established using the full spectral range, which had the best prediction abilities with $\mathrm{R}_{\mathrm{cv}}{ }^{2}$ of 0.9124 , $\mathrm{R}_{\mathrm{p}}^{2}$ of 0.8816 , RMSECV of 1.5889 , and RMSEP of 1.7719 , respectively. After the optimum wavelengths which is closely related to the TVB-N content of beef samples was obtained using the competitive adaptive reweighted (CARS) algorithm, a new PLSR model was established using the optimum wavelengths, which had outstanding prediction abilities with $\mathrm{R}_{\mathrm{cv}}{ }^{2}$ of $0.9235, \mathrm{R}_{\mathrm{p}}{ }^{2}$ of 0.9241 , RMSECV of 1.4881, and RMSEP of 1.4882, respectively.The study showed that HSI is a powerful technique to predict the TVB-N content in beef by a nondestructive way.
\end{abstract}

Keywords: beef, TVB-N content, hyperspectral imaging, data processing methods

\section{Introduction}

Due to the role of enzymes, microorganisms and other functions, beef is prone to spoilage during storage, transportation and processing. spoiled beef not only can not meet the needs of people's taste and nutrition, but also endanger people's health, cause disease. Therefore, the detection of beef freshness has important significance in public health.

Total volatile basic-nitrogen (TVB-N) is a substance produced in the process of beef spoilage, and its content in beef was proportional to the extent of beef spoilage. So it is an important index to evaluate the degree of beef freshness[1]. At present, the detection of beef freshness mainly includes sensory detection, physical and chemical detection, microbial detection, and so on[2-6]. However, these traditional detection methods are not conducive to the rapid detection of beef freshness in the product circulation.

With the development of sicence and technology, hyperspectral imaging (HSI) has been a very powerful tool for the nondestructive detection of agricultural and livestock products. There are also some applications of HSI in the measurement of meat food characteristics, such as tenderness, water-holding, marbling level and so on[7-9]. However, the studies on the use of HSI to evaluated the TVB-N content in beef were only a very few. In this study, nondestructive determination of TVB$\mathrm{N}$ content in beef using HSI technique will be evaluated. Furthermore some data processing methods, such as

\footnotetext{
a Corresponding author:lugan81@163.com
}

partition of sample set, spectral pretreatment, and the optimum wavelength selection, will be discussed to achieve the optimal predicting results.

\section{Experiments and Methods}

\subsection{Experimental samples}

The beef samples was collected from Wuhan Guoqiang poultry wholesale center in Hubei, China. After 24h postmortem, the muscles of longissimus dorsi were dissected from Huangpi cattle and Enshi mountain buffalo carcasses. Then the muscles were packaged and transported to the laboratory to be cut into several samples. A total of 168 beef samples were prepared, including 88 Huangpi cattle beef samples and 84 Enshi mountain buffalo beef samples numbered from 1 to 80 , respectively. Each sample was $8 \mathrm{~cm} * 8 \mathrm{~cm} * 4 \mathrm{~cm} * 3.5 \mathrm{~cm}$ (length*width*thickness) chops, with sealed bag packaging and placed in the biochemical incubator at $0^{\circ} \mathrm{C}$ $\sim 4{ }^{\circ} \mathrm{C}$.All the samples were measured in numerical sequence within 12 days, and 7 Huangpi cattle beef samples and 7 Enshi mountain buffalo beef samples were measured every 24 hours. 


\subsection{Experimental equipment}

In this study, the HSI system used for acquiring the hyperspectral images of the beef samples was the Headwall Hyperspec hyperspectral imaging system developed by Headwall Photonics, Inc. The HSI system is mainly composed of a hyperspectral imager (VNIR Concentric, Headwall photonics, USA), an illumination source, a CCD camera with an objective lens (Schneider Optics, USA), a sample mobile station (Andor Technology Inc, USA), and a computer with a SDKs control platform which was developed based on LabVIEW software.

To measure the reference values of TVB-N content of beef samples, standard diffusion dish and microburette were required and reagents such as saturated solution of potassium carbonate, water soluble gum, absorption liquid, mixed indicator solution and standard titration solution of hydrochloric acid were needed.

\subsection{Acquisition of hyperspectral data}

Before the HSI system was used to acquire the hyperspectral data of the beef samples, it was turned on for preheating thirty minutes to ensure a stable performance. Then the interface of the SDKs control platform was opened and the parameters of the spectrometer were set as follows: wavelength range of $400 \sim 1000 \mathrm{~nm}$, resolution of $0.8 \mathrm{~nm}$, exposure time of $5 \mathrm{~ms}$. And the parameters of the sample mobile station were set as follows: step distance of $110 \mathrm{~mm}$, object distance of $650 \mathrm{~mm}$, and line scan length of $90 \mathrm{~mm}$.

In order to eliminate the effect of white and black background on the hyperspectral images, the correction of black and white board was needed. Firstly, The blackboard image was obtained by the way of closing the lens cap and the whiteboard image was obtained by collecting standard white board image. Then the calibration image was calculated according to formula 1.

$$
I=\frac{I_{0}-I_{b}}{I_{w}-I_{b}}
$$

where $I$ is the calibrated image, $I_{0}$ is the original image, $I_{w}$ is the whiteboard image and $I_{b}$ is the blackboard image. After the black and white board calibration, the hyperspectral data of all the beef samples were acquired with the hyperspectral imaging system and saved in the computer.

\subsection{Measurement of the TVB-N content}

After hyperspectral data acquisition, the reference value of TVB-N content in beef sample was measured by the microdiffusion method according to the Chinese standard GB/T 5009.44-2003[10].The statistical results of TVB-N content of the 168 beef samples are as follows: the minimum of $9.80 \mathrm{mg} / 100 \mathrm{~g}$, the maximum of 32.44 $\mathrm{mg} / 100 \mathrm{~g}$, the mean of $18.0093 \mathrm{mg} / 100 \mathrm{~g}$, and the standard deviation of $5.6132 \mathrm{mg} / 100 \mathrm{~g}$.

\subsection{Data processing methods}

In this study, some data processing methods such as abnormal sample detection, spectral extraction, partition of sample set, spectral pretreatment, model establishment, optimum wavelength selection were discussed. The software ENVI4.7 was utilized to extract spectra from the hyperspectral image of the beef samples, and the software MATLAB R2010a was utilized to the rest data processing steps.

As is known that the hyperspectral data of each sample contain rich information including space and spectral information. There is an image in each wavelength and there are many pixels in each image. When the spectra of each beef sample were extract, a region of interest (ROI) included many pixels (about 5000) was selected, then the spectra of the pixels in the ROI were extracted and the average spectrum was calculated representing the spectrum of this beef sample. At the same way, all the spectra of the beef samples were obtained.The extracted spectral data from all the beef samples were organized in a matrix $(\mathrm{X})$, where the rows denote the number of samples (168 beef samples) and the columns represent the number of variables (753 wavelengths).

Abnormal samples will typically change the distribution trend of the overall data,thus affecting the accuracy of the calibration model. It is very necessary to detect and remove the abnormal samples before the calibration model is built. In this study, abnormal beef samples were detected by using the Monte Carlo sampling algorithm(MCS) [11].

Selection of the calibration and prediction sets may also affect the prediction performance of the model. In this study, 5 kinds of sample set partition methods, such as sample set partitioning based on joint $\mathrm{X}-\mathrm{Y}$ distances (SPXY) [12], Kennard-Stone(KS) [13], random sampling (RS), duplex, CG, were compared and discussed.

Spectral data pretreatment may have effects on the calibration model too, so some spectral pretreatment methods were compared here. For example, mean centering(MC), autoscale (AS), multiplicative scattering correction (MSC), standard normalized variate (SNV), first derivative (FD), second derivative (SD), orthogonal signal correction (OSC) and normalization were discussed.

Hyperspectral data exhibits inter-band correlation and multi-colinearity, which will lead to data redundancy and convergence instability in the calibration model. Wavelength selection have some advantage in solving above problem, because it can effectively prevent the repetition of information or redundancies by picking out the optimum wavelengths. The competitive adaptive reweighted sampling (CARS) algorithm [14] is excellent in selecting optimum wavelengths from the full spectral range, so it was utilized to select the optimum hyperspectral wavelengths for predicting the TVB-N content in beef. 


\subsection{Model performance parameters}

Model performance was evaluated according to the values of four parameters like the coefficient of determination $\left(\mathrm{R}_{\mathrm{cv}}{ }^{2}\right.$ and $\left.\mathrm{R}_{\mathrm{p}}{ }^{2}\right)$, root mean square error estimated by cross validation (RMSECV), and root mean square error estimated by prediction (RMSEP). The $\mathrm{R}_{\mathrm{cv}}{ }^{2}$ and $\mathrm{R}_{\mathrm{p}}{ }^{2}$ are higher, and the RMSECV and RMSEP are lower, the performance of the corresponding model is better.

\section{Results and Discussion}

\subsection{Spectral profiles}

The reflectance spectra extracted from the hyperspectral images of the 168 samples of tested beef are shown in Figure 1 .

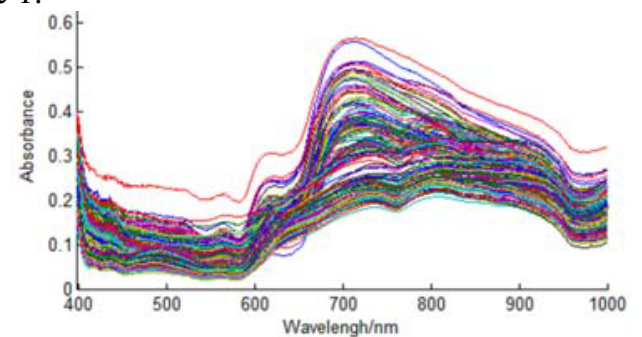

Figure1. Reflectance spectra of the samples of beef

\subsection{Abnormal beef samples}

The abnormal beef samples were detected by MCS algorithm, and the results are shown in Fig.2.

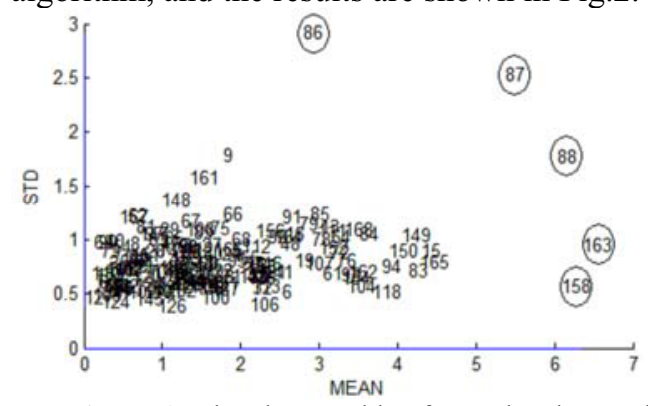

Figure 2. The abnormal beef samples detected by MCS

Figure2 indicates that sample no.86, no.87, no.88, no. 158 , and sample no. 163 were abnormal. After deleting the five abnormal samples, 163 normal samples were obtained for the subsequent analysis.

\subsection{The beef sample set partition}

The beef sample set was parted by KS, SPXY, RS, duplex, and $\mathrm{CG}$, respectively. At last, 123 samples were put into the calibration set, and 40 samples were put into the test set. The statistical results of the calibration set and the test set are shown in Table 1.

Table 1 indicates that the RS and Duplex algorithm only put the minimum of TVB-N content into the calibration set, whereas KS, SPXY, and CG algorithm put the minimum and maximum of TVB-N content all into the calibration set.Table 1 also indicates that the mean value 17.6931 of the calibration set and the mean value 17.5910 of the test set obtained by CG algorithm were all closest to the mean value 17.6680 of all samples. This shows that the calibration set and the test set obtained by $\mathrm{CG}$ were well-distributed. So CG was choosed as the best method to part beef samples.

\subsection{The full spectral range PLSR models}

The results of the PLSR models which were built using the full spectral range are shown in Table 2.

Table 2 indicates that, after the spectra were preprocessed by SNV combined with AS, the PLSR model built using the full spectral range was the best, with $\mathrm{R}_{\mathrm{cv}}{ }^{2}$ of $0.9124, \mathrm{R}_{\mathrm{p}}{ }^{2}$ of 0.8816 , RMSECV of 1.5889 and RMSEP of 1.7719. Obviously, SNV combined with AS was the best spectral preprocessing method when the PLSR model for predicting the TVB-N content in beef was built using the full spectral range.

\subsection{Selection of optimum wavelengths}

By CARS algorithm, 54 optimum wavelengths were selected. The distribution is shown in Figure 3.

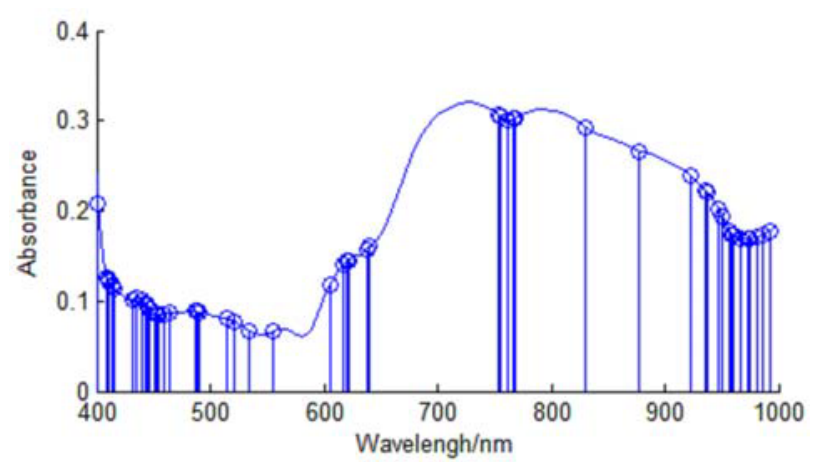

Figure 3. Distribution of the optimum wavelengths selected by CARS

\subsection{PLSR models using optimum wavelengths}

New PLSR models are built by using the optimum wavelengths and the results are shown in Table 3.

Table 3 shows that, after the spectra were preprocessed by OSC combined with MC, the PLSR model built using the optimum wavelengths was the best, with $\mathrm{R}_{\mathrm{cv}}{ }^{2}$ of $0.9235, \mathrm{R}_{\mathrm{p}}{ }^{2}$ of 0.9241 , RMSECV of 1.4881 , and RMSEP of 1.4982, respectively. Table 2 and Table 3 also indicate that, the performance of the new PLSR model was better than that of the PLSR model built using the full spectral range.Futhermore, more than $92.8 \%$ of the wavelengths were eliminated (i.e., 54 vs. 753). All above results indicated that the CARS algorithm was efficient in selecting the beef sample optimum wavelength. 
Table 1. Statistical results for the TVB-N content $(\mathrm{mg} / 100 \mathrm{~g})$ of beef samples

\begin{tabular}{cccccccc}
\hline \multirow{2}{*}{$\begin{array}{c}\text { Sample partition } \\
\text { method }\end{array}$} & \multicolumn{3}{c}{ The calibration set } & & \multicolumn{3}{c}{ The test set } \\
\cline { 2 - 4 } \cline { 6 - 8 } \cline { 6 - 8 } & Range & Mean & $\begin{array}{c}\text { Standard } \\
\text { deviation }\end{array}$ & & Range & Mean & $\begin{array}{c}\text { Standard } \\
\text { deviation }\end{array}$ \\
\hline KS & $9.80 \sim 32.4$ & 17.4663 & 5.0952 & & $10.49 \sim 30.89$ & 18.2885 & 6.0168 \\
SPXY & $9.80 \sim 32.4$ & 17.9180 & 5.2825 & & $10.49 \sim 31.70$ & 16.8993 & 5.4626 \\
RS & $9.80 \sim 30.8$ & 17.1209 & 4.9688 & & $11.18 \sim 32.44$ & 19.1949 & 6.0264 \\
Duplex & $9.80 \sim 31.7$ & 17.4644 & 5.3128 & & $10.49 \sim 32.44$ & 18.2942 & 5.3950 \\
CG & $9.80 \sim 32.4$ & 17.6931 & 5.3912 & & $10.50 \sim 30.89$ & 17.5910 & 5.1968 \\
\hline
\end{tabular}

Table 2. Results of PLSR models established using the full spectral range after different spectral pre-treatments

\begin{tabular}{cccccc}
\hline $\begin{array}{c}\text { Spectral pretreatment } \\
\text { method }\end{array}$ & $\begin{array}{c}\text { Principal component } \\
\text { numbers }\end{array}$ & $\mathrm{R}_{\mathrm{cv}}{ }^{2}$ & $\mathrm{RMSECV}$ & $\mathrm{R}_{\mathrm{p}}{ }^{2}$ & $\mathrm{RMSEP}$ \\
\hline $\mathrm{MC}$ & 20 & 0.8811 & 1.8576 & 0.8278 & 2.1369 \\
$\mathrm{AS}$ & 18 & 0.8849 & 1.8265 & 0.8340 & 2.0998 \\
$\mathrm{MSC}+\mathrm{AS}$ & 16 & 0.9112 & 1.5997 & 0.8736 & 1.8411 \\
$\mathrm{SNV}+\mathrm{AS}$ & $\mathbf{1 7}$ & $\mathbf{0 . 9 1 2 4}$ & $\mathbf{1 . 5 8 8 9}$ & $\mathbf{0 . 8 8 1 6}$ & $\mathbf{1 . 7 7 1 9}$ \\
FD+AS & 20 & 0.8533 & 2.0810 & 0.7802 & 2.4577 \\
$\mathrm{SD}+\mathrm{AS}$ & 15 & 0.9073 & 1.6352 & 0.8439 & 2.0435 \\
OSC+AS & 15 & 0.8813 & 1.8540 & 0.8270 & 2.1612 \\
Normalize+AS & 19 & 0.9123 & 1.5910 & 0.8504 & 1.9857 \\
\hline
\end{tabular}

Table 3. Results of the PLSR models established based on the selected wavelengths after different spectral pre-treatments

\begin{tabular}{cccccc}
\hline $\begin{array}{c}\text { Spectral pretreatment } \\
\text { method }\end{array}$ & $\begin{array}{c}\text { Principal component } \\
\text { numbers }\end{array}$ & $\mathrm{R}_{\mathrm{cv}}{ }^{2}$ & RMSECV & $\mathrm{R}_{\mathrm{p}}{ }^{2}$ & $\mathrm{RMSEP}^{2}$ \\
\hline none & 16 & 0.9008 & 1.6959 & 0.9137 & 1.5964 \\
MC & 16 & 0.9225 & 1.4969 & 0.9233 & 1.5010 \\
AS & 16 & 0.9190 & 1.5319 & 0.9097 & 1.6048 \\
MSC+MC & 14 & 0.9150 & 1.5679 & 0.9324 & 1.4537 \\
SNV+MC & 15 & 0.9165 & 1.5550 & 0.9326 & 1.4440 \\
OSC+MC & $\mathbf{1 3}$ & $\mathbf{0 . 9 2 3 5}$ & $\mathbf{1 . 4 8 8 1}$ & $\mathbf{0 . 9 2 4 1}$ & $\mathbf{1 . 4 9 8 2}$ \\
Normalize+MC & 15 & 0.9099 & 1.6166 & 0.9203 & 1.4871 \\
\hline
\end{tabular}

\section{Conclusions}

This study aimed to investigate the aptitude of HSI technique in predicting the TVB-N content in beef. The PLSR model for predicting TVB-N content in beef was built using the full range spectra and optimized by using the spectra at the optimum wavelengths. Different data processing methods were disscused. The CG algorithm was identified as the best sample set division method for beef samples, and SNV combined with AS was determined as the best spectral preprocessing method for the PLSR model built using the full spectral range, which resulted in $R_{c v}{ }^{2}$ of $0.9124, R_{p}{ }^{2}$ of 0.8816 , RMSECV of 1.5889 , and RMSEP of 1.7719 , respectively. The optimum wavelengths were selected by CARS algorithm. Out of 753 wavelengths, only 54 optimum wavelengths were selected to develop a new PLSR prediction model, which performed effectively, with $R_{c v}{ }^{2}$ of $0.9235, R_{p}{ }^{2}$ of 0.9241 , RMSECV of 1.4881 , and RMSEP of 1.4982 , after the spectra were preprocessed by OSC combined with MC. All the results indicated that HSI system is a potential technique for rapid and non-destructive prediction of TVB-N content in beef.

\section{Acknowledgment}

Fund for this research was provided by the project 2662015QC024 supported by the Fundamental Research funds for the Central Universities.

\section{References}

1.Y.Q Lin, Z.S. Fang . Meat Research, 25,62-65.( 2011).

2. Z. Zhang, J. Tong, D.H. Chen, Y.B. Lan. Journal of Bionic Engineering, 5, 67-73(2008).

3. X. Hong, J. Wang, Z. Hai. Sensors and Actuators B: Chemical, 161,381-389(2012).

4. Z.B. Shi, Y.Y. Tong, D.H. Chen, Y. Li. Transactions of the Chinese Society for Agricultural Machinery, 40,184-188(2009).

5. D.H. Noh, S.H. Chung, S.J. Choi, S.J. Hur. Food Control, 22,133-136(2011).

6. F. Galgano, F. Favati, M. Bonadio, V. Lorusso, P. Romano. Food Research International, 42, 11471152(2009). 
7. G.Elmasry, D.W. Sun, P. Allen. Food Research International, 44,2624-2633(2011).

8. G.Elmasry, D.W. Sun, P. Allen. Journal of Food Engineering, 110,127-140(2012).

9. Y.K. Peng, J. Zhang, W. Wang, Y.Y. Li, J.H. Wu, H. Huang, X.D. Gao, W.K. Jiang. Journal of Food Engineering, 102,163-169(2011).

10. National Standard of the People's Republic of China, Method for Analysis of Hygienic Standard of Meat and Meat Products, GB/T 5009.44-2003 (2003).

11. D.S. Cao, Y.Z. Liang, Q.S. Xu, H.D. Li, X. Chen. Journal of Computational Chemistry, 31,592602(2010).

12. R.K.H. Galvao, M.C.U Araujo, G.E. Jose, M.J.C. Pontes, E.C. Silva, T.C.B. Saldanha. Talanta, 67,736$740(2005)$.

13. W. Wu, B. Walczak, D.L. Massart, S. Heuerding, F Erni, I.R. Last, K.A. Prebble. Chemometrics \& Intelligent Laboratory Systems, 33,35- 46(1996).

14. H.D. Li, Y.Z. Liang, Q.S. Xu, D.S. Cao. Analytica Chimica Acta, 648,77-84(2009). 